\title{
Minimally Invasive Lymphadenectomy for Biliary Tumors: Stepwise Progress
}

\author{
Jason W. Denbo, MD, and Schelomo Marmour, MPH, PhD, Eric H. Jensen, MD \\ Division of Surgical Oncology, Department of Surgery, University of Minnesota Medical School, Minneapolis, MN
}

A recent issue of Annals of Surgical Oncology contains a study that reports the perioperative and long-term outcomes of laparoscopic lymphadenectomy for biliary tract tumors (intrahepatic cholangiocarcinoma and gallbladder cancer). ${ }^{1}$ Ratti et al. used propensity score matching to compare laparoscopic and open lymphadenectomy for biliary tract tumors, and attempted to isolate the lymphadenectomy-related outcomes from the concomitant hepatobiliary resection. ${ }^{1}$ They reported lower overall and lymphadenectomy-related morbidity in the laparoscopic group, while the number of retrieved lymph nodes and long-term oncologic outcomes were similar between groups. ${ }^{1}$ The authors, who are true experts in the field, should be commended for their efforts to push minimally invasive techniques forward in hepatobiliary surgery.

Propensity score matching and instrumental variables are methodologies used to address selection bias, and are commonly encountered in surgical observation studies. ${ }^{2}$ The validity of these methods depends on which variables are used to predict the propensity score. Although propensity score matching can assist with the statistical control of known factors, it does not control for unknown factors or factors not included in the matching process. In this study, eight variables were selected for matching: age, American Society of Anesthesiologists (ASA) score, diagnosis, underlying liver disease, radiological nodal status, nodularity, tumor dimensions, $\mathrm{T}$ stage, and extent of hepatectomy. The chosen variables are important to determine whether the patient is a surgical candidate and to define the magnitude of the operation, but, with the

(C) Society of Surgical Oncology 2019

First Received: 15 January 2019;

Published Online: 27 March 2019

J. W. Denbo, MD

e-mail: denbo007@umn.edu exception of the radiological nodal status, have little to do with a laparoscopic lymphadenectomy. Variables that are perhaps more important to the laparoscopic lymphadenectomy component of the procedure, albeit more difficult to quantify, such as presence of vascular and biliary anatomic variants, surgeon experience, and laparoscopic hepatobiliary surgical volume, were not included in the matching process. Omission of confounding variables such as these from a propensity score analysis may lead to significantly biased results. ${ }^{3}$ Another common pitfall of propensity score analyses of surgical procedures is the accounting of crossover procedures (i.e. a procedure that was started as laparoscopic and converted to open). ${ }^{2}$ Obviously, crossover procedures should be analyzed with intent-to-treat principles, but most commonly this is not the case in retrospective surgical observational studies. Selection of variables for propensity score matching, and accounting for crossover procedures, are only a couple of many methodological issues that must be considered and reported in a propensity score analysis. It is imperative that an individual with statistical expertise be involved with the construction and design of a propensity score analysis.

Expert hepatobiliary surgeons have detailed knowledge of hepatobiliary anatomy, extensive surgical experience, and sound clinical judgment. Only about $60 \%$ of patients have 'normal' (type I) hepatic arterial anatomy as is frequently reproduced in anatomy texts such as Netter; 10 different variations of arterial anatomy have been described (i.e. replaced right hepatic artery, accessory left, etc.). ${ }^{4}$ The knowledge and recognition of these variations is essential for safe hepatobiliary surgery. Most variations in biliary and portal venous anatomy are encountered in an intrahepatic and perihilar location and are less relevant to a hepatoduodenal ligament lymphadenectomy, but failure to appreciate these variations during hepatobiliary resections can result in catastrophic complications. Lymphatic drainage of the gallbladder, as initially described by Ito et al., 
follows three pathways through the hepatoduodenal and gastrohepatic ligaments to terminate in the retropancreatic (main pathway), celiac, and mesenteric nodes. ${ }^{5}$ The liver has deep and superficial lymphatic pathways that can drain to phrenic or mediastinal nodes via the hepatic veins and caval hiatus or to hilar nodes following the portal branches. The recent technical advances in cross-sectional imaging allow the expert hepatobiliary surgeon to appreciate anatomic variations and nuances preoperatively and decide on the most appropriate operative approach/technique.

Minimally invasive abdominal surgery has been widely accepted and implemented globally, although the adoption of minimally invasive hepatectomy has proceeded at a much slower pace. The location of the liver and biliary tract, intra- and extrahepatic anatomic complexity, proximity of large blood vessels, paucity of adequate instrumentation and techniques, and risk of major morbidity and mortality have led to a more cautious adoption. The acceptance and implementation of minimally invasive hepatobiliary surgery has been driven by small groups of international experts in the field, many of whom have espoused a stepwise evolution or implementation. ${ }^{6,7}$ Stepwise adoption of minimally invasive surgical techniques has resulted in low conversion, morbidity, and mortality rates, even though case complexity has increased. ${ }^{6,7}$ These groups of surgeons have continued to stretch the boundaries of minimally invasive techniques, but, on a national scale, only a minority of hepatobiliary cancers are treated in this fashion. A recent National Surgical Quality Improvement Program (NSQIP) study analyzed all hepatectomies performed in 2014 and reported that only 15.8\% of primary hepatobiliary cancers underwent a minimally invasive resection, while the proportion of minimally invasive procedures was somewhat higher for secondary malignancies and benign lesions. ${ }^{8}$ Clearly, there is plenty of room for adoption and implementation of minimally invasive procedures for hepatobiliary malignancies.

In this study by Ratti et al., one of the unspoken prerequisites for performing a laparoscopic lymphadenectomy for biliary tract tumors was previous mastery of minimally invasive hepatectomy, which significantly limits the applicability of the short- and long-term outcomes to the typical hepatobiliary surgeon, although the results of this study do suggest that in appropriately selected patients, expert minimally invasive hepatobiliary surgeons can perform a laparoscopic lymphadenectomy without an increased rate of associated morbidity. Therefore, for expert hepatobiliary surgeons who have been reluctant to perform minimally invasive resections for hepatobiliary cancers due to the associated lymphadenectomy, maybe it is time to take the next step.

Although propensity score analysis is a powerful statistical tool, it is imperative to be cognizant and critical of the methodological limitations of studies that utilize this technique. Lastly, the future of oncologic hepatobiliary surgery lies in safe stepwise adoption and implementation of minimally invasive techniques and therapies.

\section{REFERENCES}

1. Ratti F, Fiorentini G, Cipriani F, Paganelli M, Catena M, Aldrighetti L. Perioperative and long-term outcomes of laparoscopic versus open lymphadenectomy for biliary tumors: a propensity-score-based, case-matched analysis. Ann Surg Oncol. 2019;26(2):564-75.

2. Lonjon G, Porcher R, Ergina P, Fouet M, Boutron I. Potential pitfalls of reporting and bias in observational studies with propensity score analysis assessing a surgical procedure: a methodological systematic review. Ann Surg. 2017;265(5):901-9.

3. Brookhart MA, Schneeweiss S, Rothman KJ, Glynn RJ, Avorn J, Sturmer T. Variable selection for propensity score models. Am J Epidemiol. 2006;163(12):1149-56.

4. Covey AM, Brody LA, Maluccio MA, Getrajdman GI, Brown KT Variant hepatic arterial anatomy revisited: digital subtraction angiography performed in 600 patients. Radiology. 2002;224(2):542-7.

5. Ito M, Mishima Y, Sato T. An anatomical study of the lymphatic drainage of the gallbladder. Surg Radiol Anat. 1991;13(2):89-104.

6. Komatsu S, Scatton O, Goumard C, et al. Development process and technical aspects of laparoscopic hepatectomy: learning curve based on 15 years of experience. $J$ Am Coll Surg. 2017;224(5):841-50.

7. van der Poel MJ, Huisman F, Busch OR, et al. Stepwise introduction of laparoscopic liver surgery: validation of guideline recommendations. HPB (Oxford). 2017;19(10):894-900.

8. Bagante F, Spolverato G, Strasberg SM, et al. Minimally invasive vs. Open hepatectomy: a comparative analysis of the national surgical quality improvement program database. J Gastrointest Surg. 2016;20(9):1608-17.

Publisher's Note Springer Nature remains neutral with regard to jurisdictional claims in published maps and institutional affiliations. 